\title{
ION BEAM SCULPTING AND MATERIALS SCIENCE WITH SINGLE NANOPORES
}

\author{
Final Report \\ 15 September 2001 - 14 September 2005 \\ J.A. Golovchenko and D. Branton \\ Harvard University \\ Cambridge, MA 02138
}

N O T I C E

This report was prepared as an account of work sponsored by the United States Government. Neither the United States nor the United States Department of Energy, nor any of their employees, nor any of their contractors, subcontractors, or their employees, makes any warranty, express or implied, or assumes any legal liability or responsibility for the accuracy, completeness, or usefulness of any information, apparatus, product or process disclosed or represents that its use would not infringe privately-owned rights.

Prepared for

THE U.S. DEPARTMENT OF ENERGY

AWARD NO. DE-FG02-01ER45922: Final Report

(no unexpended funds) 


\section{A. SUMMARY OF PROGRESS - 15 September 2001 - 14 September 2005}

\section{Ion Beam Sculpting and Nanopores}

a) Nanopore studies. During this past funding period we have completed a fundamental study of the ion beam sculpting process in silicon dioxide and silicon nitride using low energy argon ions as outlined in our proposal. The study was designed to experimentally explore and isolate the phenomena of materials flow after the ion beam that initiates mass flow into the nanoscale pore is turned off. The experiments consisted of systematic studies of the formation of a nanopore using ion beam sculpting with carefully crafted pulsed ion beams. In this work we have clearly demonstrated that significant mass transport occurs on nanometer length scales over time periods as long as a second after the stimulating ion beam is extinguished. The work also demonstrated for the first time that practical ion beam sculpting parameters can be determined for the technologically important material $\mathrm{SiO}_{2}$ as well as for the previously demonstrated low stress $\mathrm{SiN}_{\mathrm{x}}$. In addition, we succeeded in presenting a model that successfully extracts pertinent ion sculpting parameters for both cases. This work is reported on in D. Stein, J. Li, J. A. Golovchenko, Ion beam Sculpting Timescales, Phys. Rev. Lett, 89: 276106-1 (2002). The above studies represent the culmination of D. Stein's PhD degree, and he moved on to a postdoctoral position at Delft.

b Ion sculpting of metals. As promised in our proposal, we have made major investments in exploring the use of the ion sculpting process for metals. This has been achieved in three stages during the last funding period. In the first, sample preparation stage a lithographic method was developed for embedding micron wide and 100 to $200 \mathrm{~nm}$ thick, copper, aluminum and platinum wires near one surface of a free standing 0.5 micron thick silicon nitride membrane. These wires were made electrically accessible for conductivity measurements by connecting them to evaporated pads on the silicon wafer that supports the nitride membrane. These samples are placed in a Focused Ion Beam (FIB) machine and a $100 \mathrm{~nm}$ gap is cut perpendicularly through the wire and all the way through the membrane, effectively creating an insulating $100 \mathrm{~nm}$ gap from one side of the wire to the other. In the second phase of the experiment, we have demonstrated that all of these metals can successfully be ion sculpted to close in the gap through local low energy ion beam exposure in the FIB. The closing phenomenon is observed ex situ with a TEM electron microscope, and until our work in the third stage of our funding, there had been no mechanism other than luck to control the final dimensions of the gap on the nanoscale. The third stage of the investigation has been the implementation of in situ electron tunneling to monitor and control the nanoscale flow of materials during the ion exposure. This has worked splendidly, particularly for platinum whose surface probably is the least sensitive to contaminants in the ion beam's vacuum chamber. This is a major advance that allows for fundamental studies of ion sculpting for metals and on geometries that are distinct from nanopores. This work has clearly demonstrated that electron tunneling can provide the nanoscale sensitivity that makes it possible to monitore and control the ion sculpting process (manuscript in preparation). 
c) High energy ion sculpting During this funding period we have also implemented a high energy ion sculpting process that uses samples prepared like those described above, but on which we use $\mathrm{MeV}$ oxygen ion beams to cause nanoscale materials flow. The mechanism involved in high energy ion induced materials flow is clearly very different from those involved for low energy exposures. As an example we have found that silicon nitride suffers virtually no induced nanoscale flows under conditions that cause very large flows in silicon dioxide. We have also succeeded to make palladium silicide metallic layers to flow but not with more common metals like aluminum. We are currently implementing a feedback tunneling experiment on our high energy accelerator for quantitative in situ nanoscale ion induced flow experiments on palladium silicide.

d) Metrology of nanopores with single wall carbon nanotube probes. In order to understand and model the complex phenomena that are responsible for ion beam sculpting, it is necessary to have a complete understanding of the morphologies that the process creates on the nanoscale. Until now we have relied on transmission electron microscopy to provide information about the diameter of nanopores formed by the process. It is also important to have quantitative information about the geometry of the pore along its longitudinal dimension both to help understand the fundamental process and because application of structures like nanopores is sensitive to this aspect of the geometry of the structure. We have perfected the growth of electrically contacted single walled carbon nanotubes on atomic force microscope cantilevers to help obtain this new information. The new cantilevers have single walled carbon nanotubes that can be tuned in length, and we are now beginning studies of nanotube geometry by threading the nanopores with nanotubes in an aqueous electrolyte solution. The study of the ionic conductance of the pore as a function of tube location in the pore will provide the needed information.

\section{Properties of Molecules Confined in Nanopores}

a) Capturing molecules in a nanopore. Using single channel recording methods and a statistical analysis of many single molecule events, we completed experiments to determine how voltage influences capture of DNA in a proteinaceous nanopore. In this system a voltage bias was used to drive charged nucleic acid molecules through a single $\sim 1.8 \mathrm{~nm}$ pore in a protein channel ( $\alpha$-hemolysin from $S$. aureus) that is incorporated in a phospholipid membrane. The system provides information about the behavior of single molecules, but in sufficient number that a statistical analysis is appropriate. Our results demonstrate that the mean time between capture events follows a simple exponential distribution, whereas the translocation times follow a unique distribution that is partly Gaussian and partly exponential. Measurements of polymer sequence effects demonstrated that translocation duration is heavily influenced by specific or non-specific purine-channel interactions. The single molecule approach we used revealed molecular interactions that can influence both capture rates and translocation velocities in a manner that enriches naive barrier crossing models.

b) Strand separation in a nanopore. We also initiated a new single molecule approach to the kinetics of strand separation in dsDNA (double stranded DNA). This approach uses the $\alpha$-hemolysin system described above to study DNA unzipping in a manner that does not require any covalent modification of the molecules being studied and is well suited to studying strand separation in short oligomers that can be synthesized with any desired sequence. Thus, our new method opens the way to detailed examinations of any sequence effects, many of which could not be carried out using naturally occurring DNA. We demonstrate that force-induced 
unzipping follows a one-dimensional kinetic pathway and use the measured kinetic parameters to infer the effective charge on DNA in the $\alpha$-hemolysin pore, a system of considerable interest for its potential biotechnological applications. This represents the first measurement of the effective charge on a molecule within the confined space of a nanopore.

c) DNA molecules and configurations in solid-state nanopores. A major advance has been achieved with the first comprehensive study of ds DNA molecules translocating through solid state nanopores during this funding period. Studies of 3 and $10 \mathrm{kbp}$ DNA have yielded fundamental information about the speeds these molecules achieve under electrophoretic drive in $10 \mathrm{~nm}$ solid state nanopores $(1 \mathrm{~cm} / \mathrm{sec}$ with $120 \mathrm{mV}$ of electrophoretic drive). This speed is deemed compatible with notions from macroscopic fluidics and ionics extrapolated into the nanoregime. More interestingly, quantized current blockades were discovered in a statistical analysis of "instantaneous" blockade levels. This led unambiguously to the interpretation of the different discrete blockade currents as corresponding to different molecule configurations in the nanopore at any given time. The simplest configurations discovered so far appear to be single molecule foldings, and multimolecule bindings. A manuscript has been submitted for publication (J. Li, M. Gershow, D. M. Stein, E. Brandin, and J. A Golovchenko, DNA Molecules and Configurations in a Solid State Nanopore, submitted for publication).

\section{Publications since last progress report}

Sauer-Budge, A.F., Nyamwanda, J.A., Lubensky, D.K. \& Branton, D. Unzipping kinetics of double-stranded DNA in a nanopore. Phys. Rev. Lett. 90, 2381011 - 2381014 (2003).

Peng, H.B., Ristroph, T.G., Schürmann, G.M., King, G.M, Yoon, J., Narayanamurti, V.\& Golovchenko, J.A. Patterned growth of single-walled carbon nanotube arrays from a vapordeposited Fe catalyst. App. Phys. Lett. 83, 4238-4240 (2003).

Li, J., Gershow, M., Stein, D., Brandin, E. \& Golovchenko, J.A. DNA molecules and configurations in a solid-state nanopore microscope. Nature Materials 2, 611-615 (2003).

Chen, P., Mitsui, T., Farmer, D.B., Golovchenko, J., Gordon, R.G. \& Branton, D. Atomic layer deposition to fine-tune the surface properties and diameters of fabricated nanopores. Nano Letters 4, 1333-1337 (2004).

Peng, H.B. \& Golovchenko, J.A. Coulomb blockade in suspended Si3N4-coated single-walled carbon nanotubes. Appl. Phys. Lett. 84,5428-5430 (2004).

Wang, H., Dunning, J.E., Huang, A.P.-H., Nyamwanda, J.A. \& Branton, D. DNA heterogeneity and phosphorylation unveiled by single-molecule electrophoresis. Proc. Natl. Acad Sci. USA 101, 13472-13477 (2004).

Stein, D.M., McMullan, C.J., Li, J.\& Golovchenko, J.A. Feedback-controlled ion beam sculpting apparatus. Review of Scientific Instruments 75, 900-905 (2004). 
Chen, P., Gu, J., Brandin, E., Kim, Y.-R., Wang, Q. \& Branton, D. Probing single DNA molecule transport using fabricated nanopores. Nano Letters 4, 2293-2298 (2004).

Ristroph, T., Goodsell, A., Golovchenko, J.A. \& Hau, L.V. Detection and quantized conductance of neutral atoms near a charged carbon nanotube. Phys. Rev. Lett. 94, 066102-1 - 066102-4 (2005).

King, G.M., Schurmann, G., Branton, D. \& Golovchenko, J.A. Nanometer patterning with ice. Nano Letters 5, 1157-1160 (2005).

Mitsui, T., D. Stein, Y.-R. Kim, D. Hoogerheide, and J.A. Golovchenko. 2006. Nanoscale volcanoes: Accretion of matter at ion-sculpted nanopores. Phys. Rev. Lett. 96, 036102-1 036102-4.

\section{Personnel associated with the program}

Prof J.A Golovchenko --PI

Prof D. Branton -- Co-PI

Peng Chen - Post Doctoral Fellow

Young-Rok Kim - Post Doctoral Fellow

Toshi Mitsui - Post Doctoral Fellow

Stella Park - Post Doctoral Fellow

Gregor Schürmann -- Post Doctoral Fellow

Hui Wang -- Post Doctoral Fellow

Marc Gershow -- Grad Student (Physics)

David Hoogerheide - Grad Student (Physics)

Mary Hughes - Grad Student (Division of Engineering and Applied Sciences)

Gavin King -- Grad Student (Physics)

Haibing Peng - Grad Student (Division of Engineering and Applied Sciences)

Christopher Russo - Grad Student (Health Sciences and Technology Program, MIT/Harvard)

Alexis Sauer-Budge - Grad Student (Biophysics)

Derek Stein -- Grad Student ( Division of Engineering and Applied Sciences) 